\title{
From Mechatronics and Cyber - MixMechatronics to Claytronics
}

\author{
Gheorghe Gheorghe
}

\begin{abstract}
The scientific paper presenting theoretical and practical research results from Mechatronics and Cyber-Mix Mechatronics Engineering applied in industrial, economical and societal environments as well as the future trends of Claytronics. Thus, the motivation of the scientific work is mainly the motivation of mechatronic and cyber-mechatronic engineering expressed by the concepts, architectures, new achievements of mechatronic and cyber-mechatronic intelligent systems for the automotive industry (already implemented at SC Automobile Renault-Dacia SA Pitesti, SC Auto Components SA Topoloveni, etc.), for measuring and controlling auto parts, moulded parts (carter, cylinder, etc.) and type of machined parts (taps, etc.).

In addition, on the basis of a study of advanced world research, the mechatronics and cyber-micromechatronics evolution towards a new science and high-tech engineering integrated in Claytronics is presented, being able to generationally revolutionize design and future engineering for the future.
\end{abstract}

Index Terms-mechatronics, cyber-mixmechatronics, atom-catom, claytronics.

\section{The Motivation OF THIS PAPER}

Research in Mechatronics and Cyber - MixMecatronics is created, developed and matured through concepts, principles and prototype and advanced intelligence systems, implemented in intelligent industrial manufacturing in Romania.

Research in Mechatronics of INCDMTM- Bucharest began well before 1991 (when it has begun institutionally and academically in Romania) when the Institute conceived, designed, developed, validated and implemented smart mechatronic systems door the industry, economy and society, and in contrast with the academic year 1991 when the field (profile) was established in the higher education in Romania, under the specializations of mechatronics and robotics.

We could say that "the achievements of this field of Mechatronics" were initiated by INCDMTM- Bucharest, long before, even during the 1980s, when several devices were delivered to the automobile industry (e.g. Renault-Dacia Pitesti), for the automotive parts industry (e.g. IPTAPA, Slatina), and so on, of "automated products" for technological processes of multi-parametric control of auto parts known as " multi-dimensional automatic control posts", "robot control multiparametric," "automatic devices for measuring, sorting and classification (with automatic elements)", auto parts, tools, drills, roller bearing, etc, without having had the name

Manuscript received September 28, 2017; revised October 28, 2017

Gheorghe Gheorghe is with Sos. Pantelimon, 6-8, 021631, Bucharest, Romania (e-mail: geocefin@yahoo.com). "mechatronic products", which was used starting with the academic year 1991 .

That year, it was developed and implemented in some Technical Universities and in the Polytechnics and Mechatronics curriculum, based on the contribution of representatives from the fields of Mechanical Engineering, Electronic Engineering, Telecommunications Engineering, and Computer Science and Automation Engineering and Automation.

Moreover, until "integrating in the field of Mechatronics", the term of "Mechatronics" there was the field of "the Fine or Precision Mechanics". So far, constitutes the technological pillar and strategic vector for the "development" of areas of smart specialization of Mechatronics "in Romania, consecrating themselves as" a real strong promoter and ensures the durability of this new industrial area "which supports science and technology and a new smart knowledge-based economy".

Research in Claytronics and Claytronic Engineering has brought the first theoretical ideas and concepts, through which, through programmable matter, it could change the face of the world.

\section{The Motivation OF MECHATRONICS AND CYBER-MECHATRONICS ENGINEERING}

In brief, Mechatronics or Engineering Mechatronics is the specialized smart field involved mainly in conceiving, designing, selecting, analysing and control integrated smart systems that combine elements of mechatronics / micro-nano-mechatronic electronics / micro-nano-electronics with computer components and / or controllers / micro-nano-computers and or micro-nano-controllers.

Therefore, Mechatronics involves elements of mechanical engineering / micro-nano-mechanical engineering, electrical engineering / micro-computer engineering nano-electrics and / micro-nano-informatics, as the progress is directly related to high-tech computer technology [1]-[3].

A block diagram of a smart system / mechatronic micro-nano-system is shown in Fig. 1.

In brief, Mechatronics or Engineering Mechatronics is the specialized smart field involved mainly in conceiving, designing, selecting, analysing and control integrated smart systems that combine elements of mechatronics / micro-nano-mechatronic electronics / micro-nano-electronics with computer components and / or controllers / micro-nano-computers and or micro-nano-controllers.

Therefore, Mechatronics involves elements of mechanical engineering / micro-nano-mechanical engineering, electrical engineering / micro-computer engineering nano-electrics and 
/ micro-nano-informatics, as the progress is directly related to high-tech computer technology [1]-[3].

A block diagram of a smart system / mechatronic micro-nano-system is shown in Fig. 1.

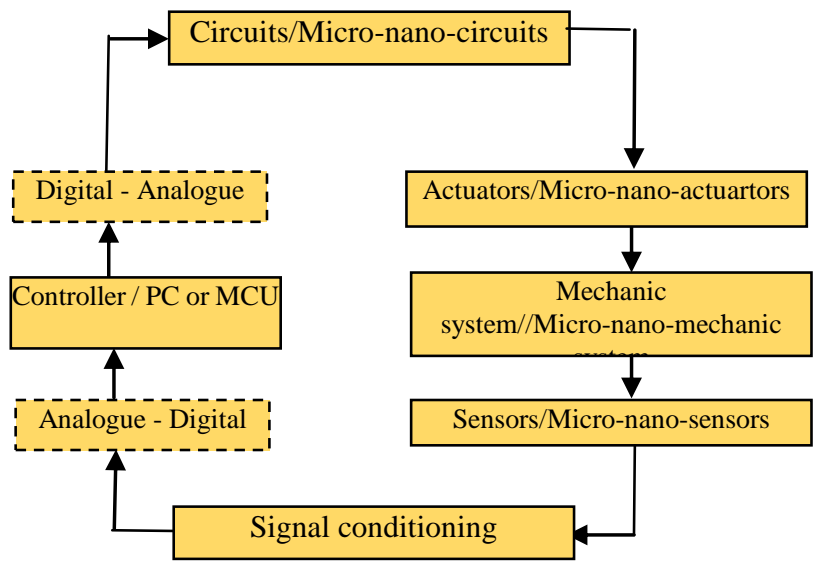

Fig. 1. Block diagram of a smart mechatronic system / micro- nano-system.

Thus, a smart mechatronic system / micro- nano-system is based on a mechanical / micro-nano-mechanical system which must be controlled; the controller needs information on system / micro-nano-systems status; this information is obtained via a variety of sensors / micro-nano-sensors (such as sensors of proximity, speed, temperature or information about travel); signals produced by the sensors / micro-nano-sensors are not in a form ready to be read by the operator and need some work on conditioning the driven signal; the conditioned signals are then converted into digital form and transmitted to the controller; the controller is the "mind" of the mechatronic systems / micro-nano-systems handling the commands and the incoming signals to generate control signals for transmission to devices / micro-nano-devices for driving the system / micro-nano-systems; the commands are obtained from a variety of supported devices / micro-nano-devices, including controls, graphical user interfaces, touch screens or pads; then control signals are transmitted to the devices / micro-nano-devices driven without using any feedback from sensors / micro-nano-sensors and thus obtaining an open loop for it to depending this requires a good calibration between entering and leaving the system / micro-nano-system and with minimum disturbance; the most common way is operating in closed loop control when the signals are sent to the actuators / micro-nano-actuators using reverse reaction from sensors / micro-nano-sensors; this mode of operation does not require calibration of information and is much better suited for handling disturbing noise; In many cases, the control signals of the devices / micro-nano-devices are first converted from the digital signal to an analogue form; amplifiers implement them as control circuits can be used to amplify the control signals sent to devices / micro-nano-devices; the actuator is the mechanism that converts electrical signals to mechanical action or movement,; the choice of a controller for a mechatronic / micro-nano-mechatronic system / micro-nano-system depends on many factors, including cost, size, ease of use as well as development and transport; many systems / micro-nano-system use personal computers (PC) with data acquisition capabilities; controllers for systems / micro-nano-systems mechatronics / micro-nano-Mechatronic are implemented using a microcontroller unit (MCU) is a device / micro-nano-devices with a single chip that includes a processor, We must highlight also other smart adaptronic systems and devices for the automobile industry, exported to specialized automotive firms in France and Portugal, as a result of the contribution of Adaptronics and Smart Adaptronics Technology:

\section{MECHATRONICS AND CYBER-MECHATRONICS ARCHITECTURE OF SMART METERING AND INTEGRATED CONTROL SYSTEMS}

Below is depicted the schematic-flowchart of mechatronic and cyber-mechatronic architecture of smart metering and integrated control systems (Fig. 6) used in smart manufacturing processes in the automotive industry, designed and produced by INCDMTM-Bucharest for SC Renault-Dacia SA Pitesti.

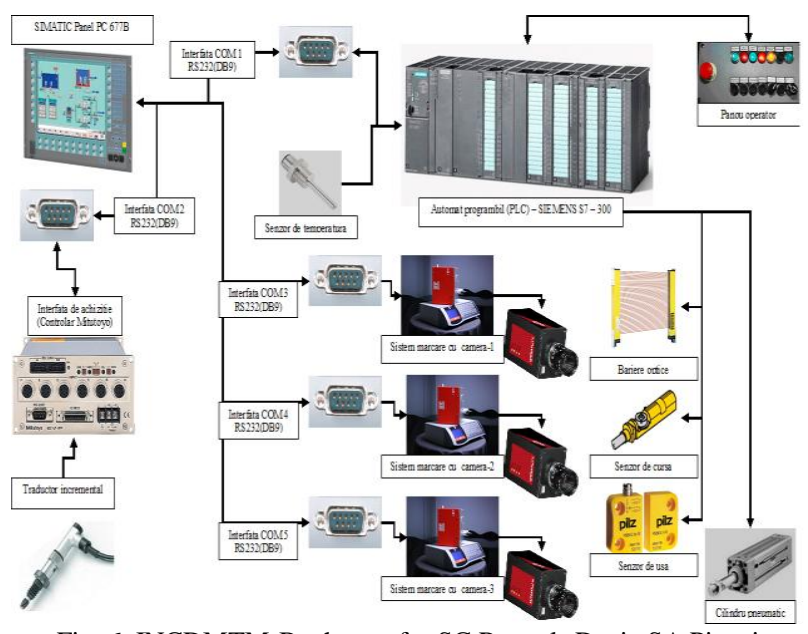

Fig. 6. INCDMTM-Bucharest for SC Renault-Dacia SA Pitesti.

Memory devices and / micro-nano-devices; microcontrollers are often used to control multiple devices / micro-nano-devices including / micro- and nano-devices available for electronic systems / micro-nano-systems for ensuring safety of vehicles; systems / micro-nano-systems that use MCU control are often called systems / micro-nano-systems built-in controls; these systems / micro-nano-systems or mechatronic control systems may be as systems / micro-nano-systems for discrete control equipment, or systems / micro-nano-systems with feedback control; in a system / micro-nano-system for discrete events, the controller controls the execution a sequence of events, while in a system / micro-nano-system with feedback control, the control controls one or more variables using sensors / micro-nano-sensors with feedback; while almost all realistic systems / micro-nano-systems involves a combination of both.

In brief, a mechatronic / micro-nano-mechatronic system / micro-nano-system integrates merged mechatronic / micro-nano-mechatronic components, electronic / micro-nano-electronic components and software implemented on a PC or MCU to produce a system / micro-nano-system which is both flexible and smart and that is able of performing complex processing of signals and data and in some cases, for a mechatronic / 
micro-nano-mechatronic system / micro-nano-system as a sole and unique means by which these systems / micro-nano-systems can operate (as control by means of magnetic bearings and the applications of control and nanometric positioning).

\section{ACHIEVEMENTS OF MECHATRONICS AND CYBER-MECHATRONICS SYSTEMS IMPLEMENTED IN INDUSTRY AND SOCIETY}

For highlighting the above idea, we present some examples of "cyber-mechatronic systems for the automotive industry," examples showing that they have already penetrated the Romanian industry (Fig. $2 \div$ Fig. 5) [4]-[6]:

1) Cyber-mechatronic device for smart adaptronic control for tightness in the following automotive part "crankcase oil" S2G Raw (Fig. 2);

2) Cyber-mechatronic device for smart adaptronic control for tightness in the following automotive part "engine cylinder head" (Fig. 3);

3) Cyber-mechatronic device for smart adaptronic control for measurement / geometry verification in the following automotive part "tappets" (Fig. 4);

4) Cyber-mechatronic machine for tightness control in the following automotive part "cylinder head - F8Q assembled" (Fig. 5).

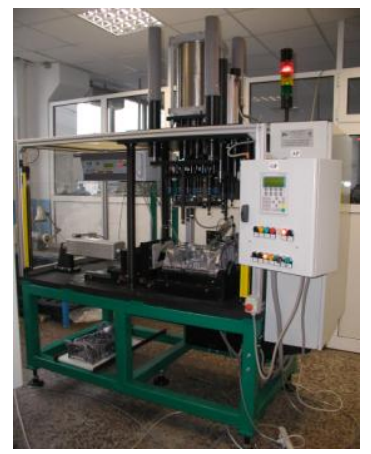

Fig. 2. Installation for controlling crankcase oil leak S2G-Raw.

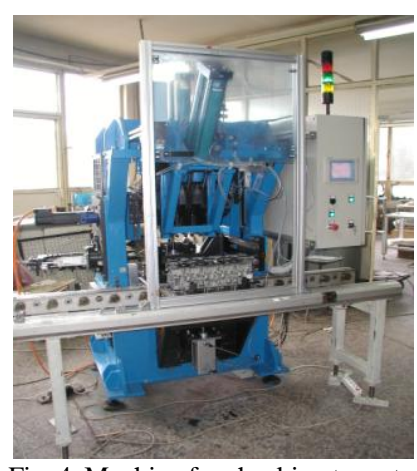

Fig. 4. Machine for checking tappets

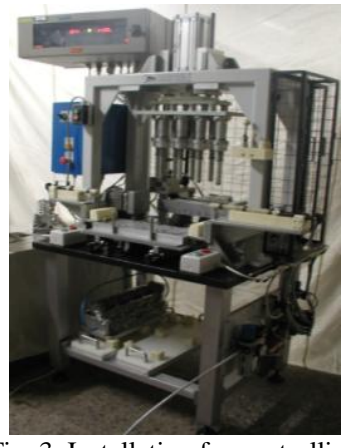

Fig. 3. Installation for controlling and marking cylinder head tightness.

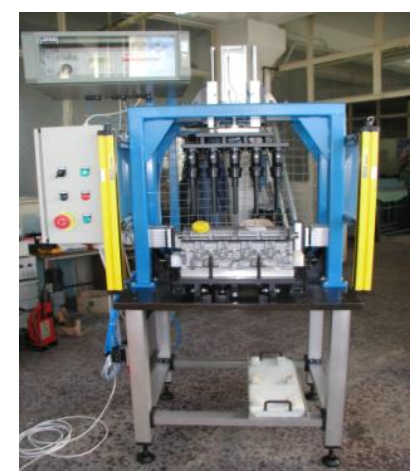

Fig. 5. Machine for checking cylinder assembled motor head tightness F8Q.

According to the figure mentioned, at the key functional and specific modules and link between, for the characterization of the automation and cybernetization process of the smart integrated control system, as follows:

-Smart mechatronic system body;

-Pneumatic driving sub-system (PLC-Siemens S7-300);
-Operator panel sub-system;

-optical barriers sub-system;

- Cycle sensor subsystem;

-Door sensor sub-system;

-Computing subsystem-Simatic Panel PC 677B;

-Interface sub-systems: - COM1 RS 232 Interface (DB9);

- COM2 RS 232 Interface (DB9);

- COM3 RS 232 Interface (DB9);

- COM4 RS 232 Interface (DB9);

- COM5 RS 232 Interface (DB9);

- Acquisition interface (Mitutoyo Controller);

- incremental encoder sub-system;

- temperature sensor sub-system;

- marking sub-systems

-with camera 1;

-with camera 2;

-with camera 3.

Smart metering and integrated control, complex mechatronic system for cyber- and integrated measuring control shown in Fig. 7.

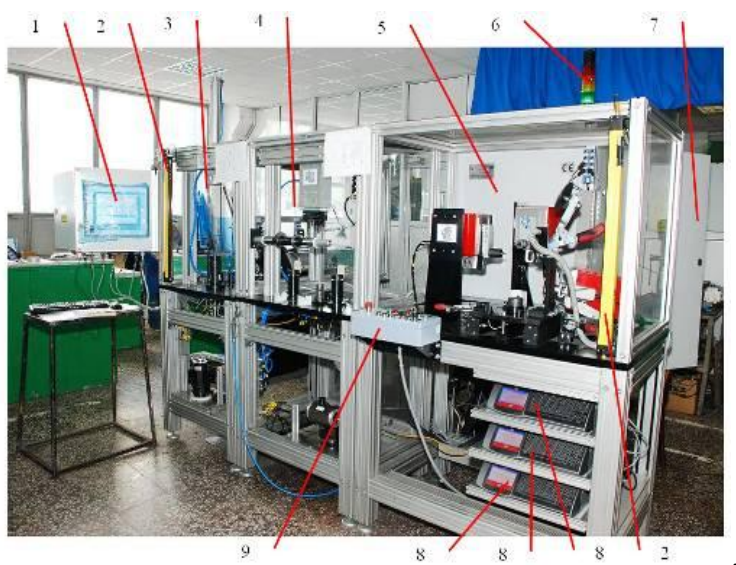

Fig. 7. Smart metering and integrated control, complex mechatronic system for cyber- and integrated measuring control.

The smart cyber-mechatronic system allows measurement of two sets of levels for the part "module return", marking the part in case the values obtained fall within the limits allowed by European regulations or norms in France and the remote-control measurement process.

The smart cyber-mechatronic system, during the technological process of automation and cybernetization, features the possibility to be monitored remotely, via teleservice, teleconfiguration and the remote control using special and pre-processing software, conditioning signal, extraction data, detecting defects and recalibration and integration Internet.

The smart cyber-mechatronic system consists of three smart stations destination for measuring $\mathrm{C}$ and dce levels, $\mathrm{K}$ and dcs levels and marking the code matrix for each of the three components of the module (body-cover-sleeve).

The smart cyber-mechatronic system has the following components:

- Measurement post 1 (3); Measurement post 2 (4); Measurement post 3 (5); PLC (7); Sensor interface (8); PC visualization applications (1); Scoring interfaces; operator panel (9); Bright column (6); Optical protection barriers (2); Post 1-piece temperature sensor; Post 2 temperature 
transducer; Ambient temperature transducer;

In the process of automation and cybernetization of measurement, control and monitoring, communication is initiated by PC-PLC (Fig. 8) and the OK of PLC indication (Fig. 9).

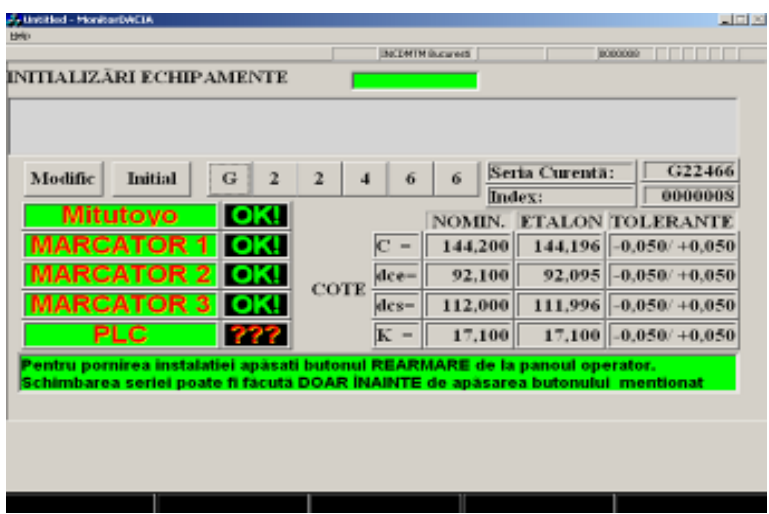

Fig. 8. PC-PLC.

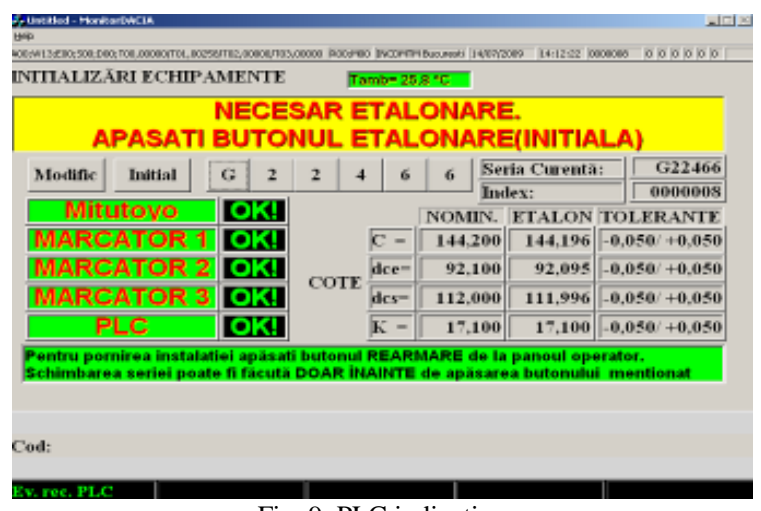

Fig. 9. PLC indication

Through active components (e.g. module IWLAN / PB PN I0; module DP / AS-i) is ensured the network Wireless connection via PROFIBUS network (via AS-i), industrial areas where the smart cyber-mechatronic system performs measuring and integrated control (Fig. 10).

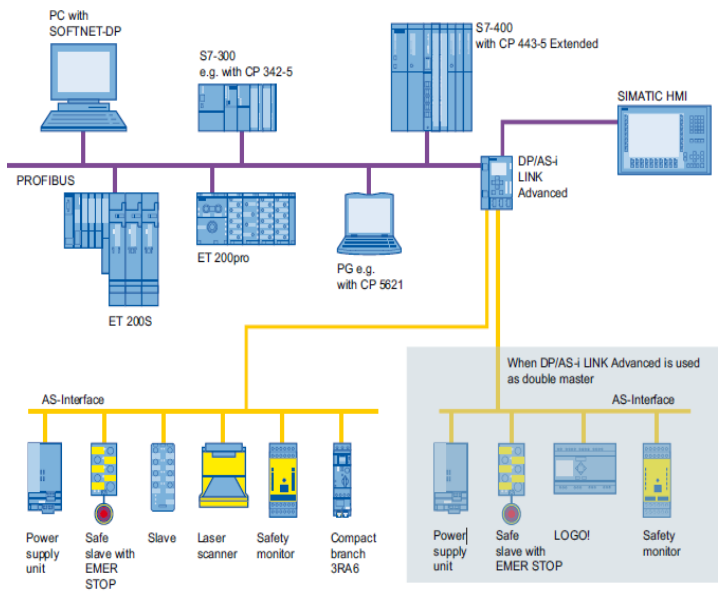

Fig. 10 Example of how to use a DP / AS-i.

Communications of all components is performed cyclically PROFIBUS DP master may require the AS-i master and performing acyclic commands, such as writing parameters, changing addresses, reading different values diagnostic.

\section{EVOLUTION OF MECHATRONICS AND CYBER-MECHATRONICS TO CLAYTRONICS}

The main objectives and motivations of the scientific paper, in response to the critical analysis of the research, are among others: - creating new concepts and original concepts of intelligent mechatronic and cyber-mixmechatronics systems; - creating new architectures and new products in advanced technological solutions integrated in intelligent industrial manufacturing in Romania; - increasing economic competitiveness through the technical and technological contribution of mechatronic and cyber- mixmechatronics engineering; - initiation of the claytronics domain and claytronics engineering creation at the university level and at the level of national research, with a view to their development in Romania and focused on the creation and development of micro-nano materials of atoms that make up the physical matter and the components of the programmable matter, synthesizing that the future of claytronics is unpredictable and technological exchanges and trends are preparing the scientific community for tomorrow; Through a scientific analysis, "atoms" form matter and "catoms" represent programmable matter composed of indivisible particles. These micro-nano-units are independent structures / micro-nano-structures, "which integrate an antenna, a processor / micro-nano-processor, sensors / micro-nano-sensors and other mechanisms / micro-nano-mechanisms" enabling them to "bind" the other "catoms"; they can be programmed in "micro-structures that create dynamic and interesting patterns in a system / micro-nano-system of programmable matter. In other words, in the future, instead of having to buy an item / micro-nano-item, one can buy only the design blueprint for the object or micro-nano-object and by using a computer, by means pf "claytronic" it can be given a three-dimensional form, taking the external challenge and obtaining programming solutions for the millions of particles associated to nano-processors working in the tandem already mentioned.

The main goal of this research and hyper-advanced cooperation is even creating a modular model or prototype of re-programmable robotic "catoms" allowing further producing and assembling them in series and the development of programming languages and software tools to control the assembly of millions of particles. Hyper-intelligent Claytronics products "will become" reality "over many more years, currently researches already creating programming language support for the "Clatronic reality" as a "Claytronic subject" that takes on the desired shape.

The claytronic atom or catom would be similar in appearance to an atom and it would be preferable to have a spherical shape, acting individually and covered by electromagnets to attach other catoms. Each catom contains a powerful processor, which has photocells on its surfaces to sense light and light emitting diodes to enable it to see and change colour (i.e. a device the size of about 1 inch in diameter and surrounded by 24 electromagnets), moving through running electromagnets, some on top of others. Claytronics can drive hardware models for micro scale devices that are much larger than the tiny modular robots.

Catoms create populate claytronic assemblies of less than 1 
millimetre in size and can cross borders pf several areas such as computer science, modular robotic systems, and nano-technological systems: planar catoms, testing the concept of movement, free effectors of motion and design for the force that creates movements and cooperative ensembles of modular robot-detents electrostatic models a new system of binding and releasing of the connection between a modular robot, a connection that creates movement and transfer power and data while employing a small factor in the strong forcestochastic catoms- that integrate the random movement with global communicated aims in plain language, to form present patterns, cooperating with other catoms of small helium to meet a set of instructions single - giant helium catoms providing a large platform to explore the relation of forces, where the electrostatic force has a greater effect than gravity on a robotic device-cubes using ratchets electrostatic order to demonstrate the functionality of a device that could be used in a system lattice-like based on self-assembly both at nano-scale, and macro-scale (see Fig. 11,12,13,14 and 15).

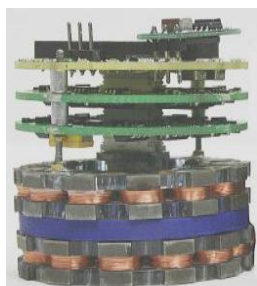

Fig. 11. Catom- V8 plain.

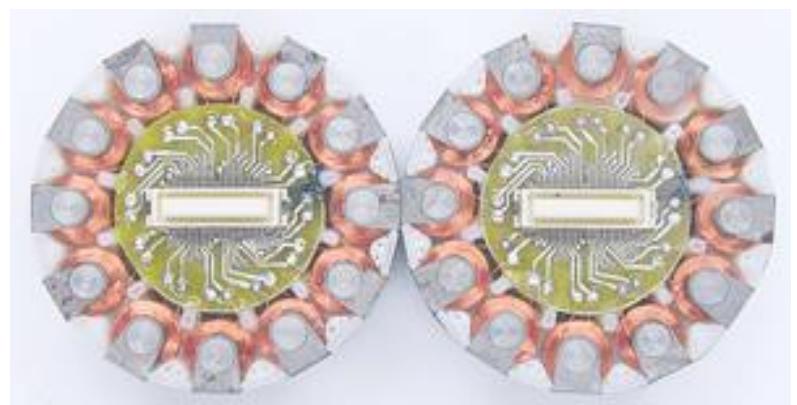

Fig. 12. Two rings on the planar magnetic V8 catom.

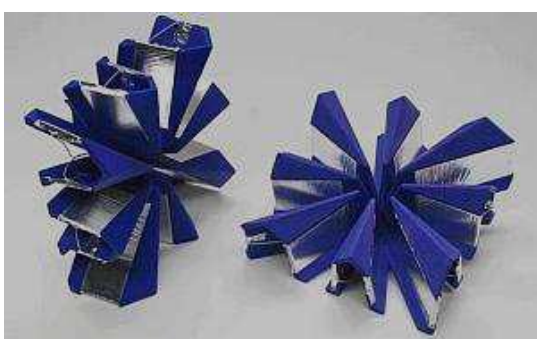

Fig. 13. Three planar LED catoms.

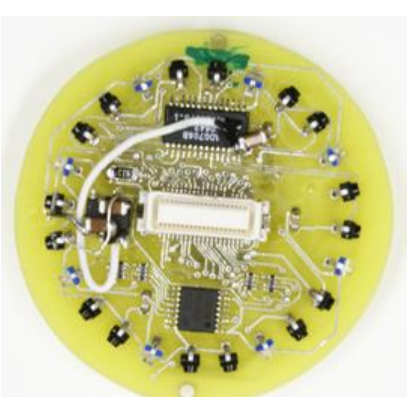

Fig. 14. Electrostatic clickings.

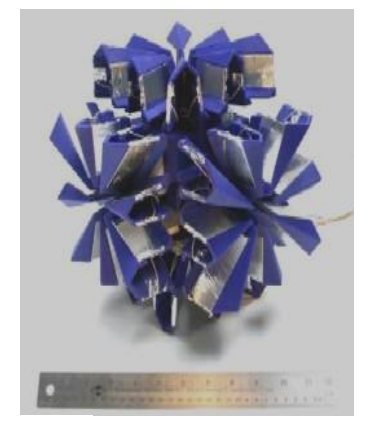

Fig. 15. Cube (for self-assembly).

Following the concept integrator, we have developed the necessary software (creation of algorithms and programming language) for organizing the millions of catom of sub-millimetre scale and to develop a complete structure of information resources in a distributed network with dense robotic knots in a Clatronic matrix, repair / eliminate mistakes integrated into the implementation of programs, creating algorithms that transform groups of catoms in the primary structure for building dynamic representations of three-dimensional dynamic creation algorithms that allow catoms to locate their positions among millions of other catoms and creating dynamic simulation for understanding the real-time performance and behaviour of Claytronic assemblies for groups of catoms.

\section{CONCLUSIONS}

In conclusion, we summarize that the future (of mechatronics and cyber-mechatronics) is unpredictable and the trends and the technological exchanges and prepare the scientific community for tomorrow.

Thus, Biotechnology, Genetics, Science of Materials, Robotics, Mechatronics and Cyber-Mix-Mechatronics and many more fields of nano-sciences and nano-technologies, fail to grow (evolve) in their way to smart planetary other new scientific discoveries.

The research results from the mechatronics and cyber-mixmechronics laboratories of INCDMTM Bucharest are obtained on various structural and informational levels, from static intelligent systems to dynamic hyper-intelligent systems with remote control, remote monitoring and remote configuration mixes, integrated in intelligent industrial applications real, industrial and laboratory feedback, contributing to the sustainability of automation and cybernetics of intelligent manufacturing processes.

Next, research for the future, still creates vectors, concepts, concepts and experimental models for Claytronics science and engineering, in order to validate new scientific discoveries.

In the near or distant future, "the Science of Claytronics" will be able to revolutionize the generative design and hyper-advanced computer engineering, telecommunications interfaces, human-computer hardware, but also new remote communication (the emergence of a new communication medium-Pario).

\section{REFERENCES}

[1] L. Davis, D. Hyland, G. Yen, and A. Das, Adaptive Neural Control for Space Structure Vibration Suppression, 1999, pp. 753-766; 
[2] L. E. A. Lee, "Cyber-physical systems: Design challenges," in Proc. International Symposium on Object/ Component/Service - Oriented Real-Time Distributed Computing, May 6, 2008.

[3] M. Busse and F. J. Wöstmann, "Intelligent cast parts - Application of adaptronic components with cast parts," in Proc. Adaptronic Congress, Göttingen, May 2006;

[4] G. I. Gheorghe, Mecatronica and Sistemele Cyber-Mecatronice, Ed. CEFIN Bucuresti, ISBN 978-606-8261-22-5, 2015;

[5] G. I. Gheorghe, Adaptronica Sistemelor Inteligente, Editura Agir, București, 2014

[6] G. I. Gheorghe, A. Constantin, and S. Dumitru, MicroNanoIngineria MEMS \& NEMS Inteligente, Editura CEFIN București, 2013.

[7] I. G. Gheorghe, A Constantin, and I. Ilie, Mechatronics and Cyber Mechatronics in Intelligent Applications from Industry and Society, Romania

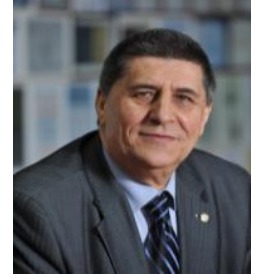

Gheorghe Gheorghe is an associate professor at the Faculty of Mechatronics Politehnica University of Bucharest, Valahia University of Targoviste an Titu Maiorescu University, Romania. He received her bachelor degree in precision mechanics from Politehnica University of Bucharest, a doctoral degree at Politehnica University of Timisoara and Doctor Honoris Causa degree from Valahia University of Targoviste, His current research includes mechatronics and cyber-mechatronics systems, measurement technique, cyber-adaptronic systems. 